\title{
Perfil de Risco Cardiovascular de Moradores de Quadras Centrais de Palmas - TO
}

\author{
Marcela Silva Menezes ${ }^{1}$, Ruth Bernardes de Lima Pereira ${ }^{2}$ \\ Danyela Martins Cerqueira ${ }^{3}$, Davyd Christian de Menezes Ferreira Leal ${ }^{4}$
}

\begin{abstract}
RESUMO
As doenças crônicas não transmissíveis são hoje a principal causa de morte no mundo. Com base na alta prevalência da mortalidade destas comorbidades e também das doenças cardiovasculares, além da carência por uma assistência que priorize a prevenção e promoção em saúde, objetivou-se, com este estudo, conhecer o perfil epidemiológico de risco cardiovascular utilizando o Escore de Framingham dos moradores assistidos por uma Equipe de Saúde da Família no munícipio de Palmas - TO. Optou-se por um estudo epidemiológico, transversal e descritivo com abordagem quantitativa. Foi realizado no munícipio de Palmas-Tocantins no período de novembro de 2016 a fevereiro de 2017. A amostra se deu por usuários do Sistema Único de Saúde e moradores do território de abrangência de uma Equipe de Estratégia Saúde da Família localizada na região central de Palmas - TO. Participaram da pesquisa 98 usuários. A média de risco absoluto foi de $6 \%$, risco considerado baixo conforme o Escore de Framingham. Cerca de $70 \%$ dos usuários estavam acima do peso. Nos usuários classificados com risco cardiovascular baixo, $65 \%$ apresentaram sobrepeso ou obesidade. O perfil de risco cardiovascular da população estudada apresentou-se satisfatório pelo Escore de Framingham, entretanto evidenciou-se a presença de outros fatores de risco associados que, ao longo do tempo, podem levar os mesmo ao adoecimento. Destaca-se a importância do cuidado integral aos indivíduos com risco absoluto baixo pelo Escore de Framingham considerando outros fatores de risco que também influenciam no surgimento de doenças crônicas não transmissíveis e doenças cardiovasculares.
\end{abstract}

Palavras-chave: Fatores de risco. Doenças cardiovasculares. Epidemiologia. Perfil de saúde.

\section{CARDIOVASCULAR RISK PROFILE OF RESIDENTS IN CENTRAL AREA OF PALMAS-TO}

\section{ABSTRACT}

Nowadays non-communicable chronic diseases are the leading cause of death worldwide. Based on the high level of mortality caused by these comorbidities and cardiovascular diseases, in addition the lack of policies that prioritizes prevention and health promotion, this study objectified to build an epidemiological profile of cardiovascular risk using the Framingham Risk Score of residents assisted by a Family Health Care Team in the city of Palmas, Tocantins. Opted for an epidemiological, cross-sectional and descriptive study with a quantitative approach. The study was conducted in the city of Palmas-Tocantins from November 2016 to February 2017. The data was gathered from users of the Unified Health System and residents of a block in the central area of Palmas. A number of 98 users participated in the study; the average absolute risk was $6 \%$, a low risk according to the Framingham Risk Score. About $70 \%$ of users were overweight. From the users classified with low cardiovascular risk, $65 \%$ were overweight or obese. The cardiovascular risk profile of the studied population was satisfactory by the Framingham Risk Score. However, evidences presented other associated risk factors that in the long term may lead those users to the illness. It is relevant to point out the importance of an overall and continuous care with the low risk individuals by the Framingham Risk Score towards other factors that also influence the rise of chronic non-communicable diseases and cardiovascular diseases.

Keywords: Risk factors. Cardiovascular diseases. Epidemiology. Health profile.

Recebido em: $2 / 3 / 2017$

Revisões requeridas em: 25/4/2018

Aceito em: 3/9/2018

\footnotetext{
Enfermeira. Residente em Saúde da Família e Comunidade. Fundação Escola de Saúde Pública de Palmas, TO. Centro Universitário Luterano de Palmas - UIbra.marcela.sime@gmail.com

${ }^{2}$ Enfermeira. Mestre em Ciências da Saúde. Orientadora. Fundação Escola de Saúde Pública de Palmas, TO. Centro Universitário Luterano de Palmas - Ulbra. ruth.bernardes@gmail.com

${ }^{3}$ Enfermeira. Residente em Saúde da Família e Comunidade. Fundação Escola de Saúde Pública de Palmas, TO. Centro Universitário Luterano de Palmas - UIbra. danyelacerqueira@gmail.com

${ }^{4}$ Enfermeiro. Especialista em Saúde da Família. Fundação Escola de Saúde Pública de Palmas, TO. dyleal@gmail.com
} 


\section{INTRODUÇÃO}

As doenças crônicas não transmissíveis (DCNTs) são hoje a principal causa de morte no mundo. Possuem grande importância para a saúde pública e estão geralmente associadas a fatores de riscos passíveis de intervenção, como tabagismo, inatividade física, hábitos alimentares inadequados e etilismo (SCHMIDT et al., 2011). Entre as DCNTs, destacam-se as doenças cardiovasculares (DCV) que, em 2008, representaram $48 \%$ das mortes por doenças crônicas e $30 \%$ das mortes totais no mesmo ano (BEATTY et al., 2012).

Em Palmas, capital do Tocantins, localizada na Região Norte do Brasil, estima-se uma população de quase 280 mil habitantes em 2016 segundo o IBGE (INSTITUTO..., 2016a). A oferta de serviços de saúde ocupa uma posição confortável no município, com cerca de $92 \%$ de cobertura (BRASIL, 2017b). O município teve as doenças do aparelho circulatório como primeira causa de óbitos, com $23 \%$, entre os anos de 2010 e 2014 (BRASIL, 2016).

Com base nestes agravantes, evidencia-se a necessidade de políticas públicas voltadas para a prevenção e controle das DCNTs, razão pela qual se popularizou a utilização dos escores de risco para triagem e classificação de risco cardiovascular da população suscetível ao adoecimento. O Escore de Framingham (EF) é um dos mais utilizados neste contexto; originou-se do Framingham Heart Study, um estudo desenvolvido em 1948, com o objetivo de identificar fatores de risco para DCV. O EF por meio do valor da pressão sistólica, a presença de diabetes, tabagismo, níveis de colesterol total, fração HDL, sexo e faixa etária, pode estimar o risco cardiovascular (RCV) de uma pessoa em até dez anos (LOTUFO, 2008).

O Ministério da Saúde recomenda, para estratificação do risco global, o EF revisado em 2005 no Manual de Capacitação dos Profissionais de Saúde da Rede Básica. Neste escore utiliza-se a fração LDL, substituindo o colesterol total (BRASIL, 2006).

O EF possibilita, além da estratificação e identificação de fatores de RCV, atuar de forma preventiva antecipando ações para evitar o adoecimento. Além disso, sua aplicação é recomendada pelo Ministério da Saúde em vários cadernos da Atenção Básica. Desta forma, o escore pode ser utilizado como um método de auxílio para direcionamento e melhor planejamento da assistência aos pacientes submetidos à avaliação (BRASIL, 2006, 2013).

A estratificação de risco com o EF, assim como outras estratégias recomendadas pelo Ministério da Saúde, permite um diagnóstico pontual e preciso para mensurar fatores de riscos e, assim, priorizar cuidados e intervenções. Pelo cálculo, é possível individualizar as ações a serem desenvolvidas, bem como, em larga escala, eleger fatores de riscos comuns a um determinado território e orientar a equipe multiprofissional no planejamento de estratégias para controle e prevenção de DCNT e DCV.

Com base na alta prevalência da mortalidade por DCV e DCNT, além da carência por uma assistência que priorize a prevenção e promoção em saúde, objetivou-se, com este estudo, conhecer o perfil epidemiológico de risco cardiovascular utilizando o EF dos moradores assistidos por uma equipe de Estratégia Saúde da Família (ESF) no munícipio de Palmas - TO.

\section{MÉTODO}

É um estudo epidemiológico, transversal e descritivo com abordagem quantitativa. Foi realizado no munícipio de Palmas - TO - no período de novembro de 2016 a fevereiro de 2017. A amostra se deu por usuários do Sistema Único de Saúde e moradores do território de abrangência de uma Equipe de ESF localizada na região central de Palmas - TO.

Este território é composto por três quadras na região central de Palmas - TO -; conta com 100\% de cobertura por Agentes Comunitários de Saúde e também da ESF, e possui amplo acesso à Unidade Saúde da Família (USF), que fica localizada dentro do território de abrangência. A população na faixa etária de 30 a 60 anos é de 1.248 habitantes.

Para cálculo da amostra, levou-se em consideração o levantamento populacional realizado pelos Agentes Comunitários de Saúde (ACS) na Programação para Gestão por Resultados na Atenção Básica (Prograb). Utilizou-se o programa OpenEpi, versão 3, considerando intervalo de confiança de $97 \%$, totalizando 69 usuários.

Os critérios de inclusão do estudo foram: idade entre 30 e 60 anos, ambos os sexos, moradores do território de abrangência da equipe, sem cardiopatia prévia e que concordasse em participar da pesquisa mediante assinatura do Termo de Consentimento Livre e Esclarecido (TCLE). Foram excluídos deste estudo os pacientes fora da faixa etária mencionada, gestantes, portadores de doença cardíaca prévia e todos os que não atenderam os demais critérios de inclusão.

Os participantes da pesquisa foram convidados por conveniência pelos agentes de saúde do território durante as visitas domiciliares e também por profissionais da equipe durante o atendimento na USF. Depois de convidados, os participantes que concordaram 
em participar da pesquisa foram encaminhados à consulta de enfermagem com os pesquisadores para verificação de dados antropométricos, pressão arterial, entrevista e solicitação de exames laboratoriais. Neste primeiro contato, os participantes foram entrevistados abordando aspectos como sedentarismo, presença de comorbidades e tabagismo.

Para este estudo, consideramos sedentário ou com inatividade física todo o indivíduo que não realizou nenhuma atividade física no tempo livre nos últimos três meses e que não fez esforços físicos no ambiente que frequenta diariamente. Para tabagismo, considerou-se qualquer indivíduo que referisse o hábito de fumar, independente da quantidade de cigarros ao dia (BRASIL, 2017a).

Os dados antropométricos verificados foram altura, peso e índice de massa corporal (IMC). Os valores do IMC até 24,9 foram classificados como adequados, acima de 25 até 29,9 , sobrepeso e acima de 30 , obesidade, sendo a última dividida em graus I, II e III (ASSOCIAÇÃO..., 2009). Verificou-se, ainda, a pressão arterial do paciente e foram solicitados exames laboratoriais: hemograma completo, lipidograma completo, glicemia de jejum, urina tipo 1, ureia e creatinina. Após esta etapa, o paciente foi agendado para consulta subsequente, quando foi calculado o risco cardiovascular utilizando o EF revisado (BRASIL, 2006).

Os princípios éticos que nortearam esta pesquisa estão contemplados na Resolução 466/12. Este estudo foi iniciado após aprovação do Comitê de Ética em Pesquisa do Centro Universitário Luterano de Palmas - TO (Ceulp - Ulbra) - sob $n^{\circ}$ de parecer 1.837.049. Todos os participantes desta pesquisa assinaram o TCLE (BRASIL, 2012). Para análise e interpretação dos dados foram utilizados gráficos e tabelas do Excel, além de análises pelo programa Epi Info.

\section{RESULTADOS E DISCUSSÃO}

O foco deste estudo foi os adultos sem cardiopatia prévia na faixa etária de 30 a 60 anos. Outras comorbidades não foram elencadas como critério com o intuito de representar a população conforme suas características, incluindo a presença ou não de outras DCNTs. Os usuários, durante a primeira consulta, foram esclarecidos quanto ao objetivo do EF e orientados quanto à importância da prevenção e promoção da saúde no âmbito das DCNTs e DCV, além dos benefícios que poderiam ter com o conhecimento a respeito do seu RCV.
Participaram da pesquisa 98 usuários, com uma perda de $28 \%$ em razão da ausência do retorno com os resultados de exames. Tal fato pode ser explicado pelo modelo de saúde centrado na doença que ainda possui grande influência na população, gerando um empecilho quando se busca a promoção e prevenção em saúde antes do aparecimento de doenças (OLIVEIRA; SILVA, 2010). Este fato influenciou diretamente na população, que não retornou para as demais análises, resultando em uma amostra final composta por 71 usuários.

Em relação ao sexo e à faixa etária, a média de idade foi de 46 anos. A média de risco absoluto do EF foi de $6 \%$, risco considerado baixo. As mulheres representaram a maioria neste estudo, com cerca de $46,4 \%$ a mais que a porcentagem de homens. Supõe-se que este resultado se deu pela maior procura das mulheres pelos serviços de saúde. De acordo com outros autores, há uma participação mais frequente da mulher, enquanto o homem, geralmente, busca o atendimento em situações agudas. Tal fato demonstra a fragilidade da assistência quando se busca efetivar intervenções preventivas (MOURA et al., 2013).

Tabela 1 - Distribuição da população estudada segundo sexo e faixa etária, Palmas - TO -, 2016

\begin{tabular}{|c|c|c|c|c|c|c|c|c|}
\hline \multicolumn{3}{|c|}{ Sexo } & \multicolumn{2}{|c|}{$n$} & \multicolumn{4}{|c|}{$\%$} \\
\hline \multicolumn{3}{|c|}{ Masculino } & \multicolumn{2}{|c|}{19} & \multicolumn{4}{|c|}{26,8} \\
\hline \multicolumn{3}{|c|}{ Feminino } & \multicolumn{2}{|c|}{52} & \multicolumn{4}{|c|}{73,2} \\
\hline \multicolumn{3}{|c|}{ Total } & \multicolumn{2}{|c|}{71} & \multicolumn{4}{|c|}{100} \\
\hline \multirow[t]{2}{*}{ Faixa Etária } & \multicolumn{2}{|c|}{$30-39$} & \multicolumn{2}{|c|}{$40-49$} & \multicolumn{2}{|c|}{$50-59$} & \multicolumn{2}{|c|}{60} \\
\hline & $\mathrm{n}$ & $\%$ & $\mathrm{n}$ & $\%$ & $\mathrm{n}$ & $\%$ & $\mathrm{n}$ & $\%$ \\
\hline Masculino & 3 & 15,8 & 7 & 36,8 & 8 & 42,1 & 1 & 5,3 \\
\hline Feminino & 17 & 32,7 & 12 & 23,1 & 23 & 44,2 & 0 & 0 \\
\hline Total & 20 & 28,2 & 19 & 26,8 & 31 & 43,7 & 1 & 1,4 \\
\hline
\end{tabular}

Fonte: Dados dos autores.

A faixa etária mais frequente foi entre 50 e 59 anos para homens e mulheres, que representou quase $44 \%$ da amostra, sem variação da taxa entre os sexos. A maior frequência da faixa etária de 50 a 59 anos pode ser explicada pela maior preocupação com a saúde associada ao envelhecimento e procura do USF com mais assiduidade. Na faixa etária menor de 50 anos, a amostra foi de $55 \%$.

Em relação ao tabagismo, a frequência encontrada foi inferior a $3 \%$ nos pesquisados, apresentando pequena diferença em relação ao Vigitel (BRASIL, 2017a), quando Palmas - TO - obteve um percentual de $4,4 \%$. Isso pode ser explicado pela tendência de- 
crescente do hábito, posto que a frequência do tabagismo tem diminuído em média 0,7 ponto percentual ao ano, demonstrando uma evolução extremamente favorável (BRASIL, 2017a).

As comorbidades apresentadas pelos participantes do estudo foram Hipertensão Arterial Sistêmica com 45\%, Diabetes Mellitus tipo 2 com quase 15\% e Depressão com $4 \%$. Neste estudo, mais de $50 \%$ de todos os usuários apresentaram alguma comorbidade prévia, e $7 \%$ destes apresentaram duas ou mais.

A presença destas DCNTs implica um aumento dos fatores de RCV associados, tanto os avaliados pelo EF quanto outros fatores independentes, como sobrepeso, obesidade e sedentarismo. A depressão, em si, não se associa diretamente ao risco cardiovascular, entretanto influencia no autocuidado e no manejo do usuário com outras DCNTs presentes, portanto pode ser um agravante que dificulta o controle destas situações clínicas (SANTOS; PINHO, 2012; PIMENTA; CALDEIRA, 2014; MADEIRA et al., 2013).

O risco baixo foi prevalente nesta população, conforme mostra a tabela a seguir:
Evidenciou-se maior frequência de riscos médio/alto em homens, com $26,4 \%$, enquanto as muIheres apresentaram uma frequência de $15,3 \%$. Os escores de riscos médio/alto foram evidenciados em cerca de $18 \%$ dos usuários. Neste grupo, a média de idade foi de 53 anos. O risco elevado (médio/alto) na faixa etária de 50 a 59 anos prediz um risco de morte importante por DCV antes dos 75,5 anos, que é a expectativa de vida do brasileiro (INSTITUTO..., 2016b).

As comorbidades e o sedentarismo apareceram em $100 \%$ da população com médio e alto risco. Estas variáveis são consideradas fator de risco isolado, que, apesar de não serem levadas em conta no $\mathrm{EF}$, apresentam influência em outros fatores avaliados, como na fração LDL e HDL, valores da pressão arterial e a presença ou não de Diabetes Mellitus (DM).

A exposição a fatores de risco surge cada vez mais cedo na rotina de vida atual, o que, de fato, resulta no surgimento antecipado das DCVs e também das DCNTS (GOMES; MOREIRA, 2014; GUERRA, 2008). Este fato reforça a importância do EF como ferramenta de estratificação e de identificação dos fatores de risco, que podem se agravar com o avançar da idade.

Tabela 2 - Distribuição de risco cardiovascular, comorbidades e sedentarismo na população estudada segundo sexo, Palmas -TO - , 2016

\begin{tabular}{ccccccccc}
\hline \multirow{2}{*}{ Sexo } & \multicolumn{2}{c}{ RCV Baixo } & \multicolumn{2}{c}{ RCV Médio } & \multicolumn{2}{c}{ RCV Alto } & \multicolumn{2}{c}{ Total } \\
\cline { 2 - 10 } & $\mathrm{n}$ & $\%$ & $\mathrm{n}$ & $\%$ & $\mathrm{n}$ & $\%$ & $\mathrm{n}$ & $\%$ \\
\hline Masculino & 14 & 73,7 & 4 & 21,1 & 1 & 5,3 & 19 & 100 \\
\hline Feminino & 44 & 84,6 & 6 & 11,5 & 2 & 3,8 & 52 & 100 \\
\hline \multirow{2}{*}{ Total } & 58 & 81,7 & 10 & 14,1 & 3 & 4,8 & 71 & 100 \\
\hline \multirow{2}{*}{ Sexo } & Comorbidades & Comorbidades & Comorbidades & \multicolumn{2}{c}{ Total } \\
\cline { 2 - 10 } & $\mathrm{n}$ & $\%$ & $\mathrm{n}$ & $\%$ & $\mathrm{n}$ & $\%$ & $\mathrm{n}$ & $\%$ \\
\hline Masculino & 7 & 36,8 & 4 & 21,1 & 1 & 5,3 & 12 & 63,2 \\
\hline Feminino & 18 & 34,6 & 6 & 11,5 & 2 & 3,8 & 25 & 48,1 \\
\hline Total & 25 & 43 & 10 & 14,1 & 3 & 4,2 & 38 & 52,1 \\
\hline & Sedentarismo & Sedentarismo & Sedentarismo & \multicolumn{2}{c}{ Total } \\
\cline { 2 - 10 } Sexo & $\mathrm{n}$ & $\%$ & $\mathrm{n}$ & $\%$ & $\mathrm{n}$ & $\%$ & $\mathrm{n}$ & $\%$ \\
\hline Masculino & 9 & 64,3 & 4 & 21,1 & 1 & 5,3 & 14 & 73,7 \\
\hline Feminino & 24 & 54,5 & 6 & 11,5 & 2 & 3,8 & 32 & 61,5 \\
\hline Total & 33 & 57 & 10 & 14,1 & 3 & 4,8 & 46 & 64,8 \\
\hline
\end{tabular}

Fonte: Dados dos autores. 
O risco baixo do $E F$, que varia entre $1 \%$ e $9 \%$ em homens e mulheres, alcançou a maior representação com mais de $80 \%$ dos usuários. Este resultado era esperado quando se considerasse a idade dos participantes, pois a variável idade possui influência gradativa no cálculo do EF. A pontuação da idade atua como fator de proteção até os 39 e 44 anos para homens e mulheres, respectivamente, muitas vezes até reduzindo o escore do usuário (BRASIL, 2006).

A análise desse dado deve ser feita de forma cuidadosa, pois, a princípio, um risco considerado baixo de desenvolver uma DCV em dez anos é interpretado apenas de forma objetiva como algo satisfatório, porém é preciso avaliar isoladamente todas as variáveis do escore. O risco absoluto baixo no EF pode mascarar outros riscos e adiar uma estratificação com risco elevado, por falta de intervenção no risco evidenciado.

Na Tabela 2 é possível verificar, por exemplo, outros fatores de riscos isolados dos usuários com baixo risco para DCV. Estes não são considerados no EF, porém tiveram valor expressivo no total pesquisado. As comorbidades apareceram em $43 \%$ dos usuários com risco baixo, enquanto $57 \%$ foram classificados como sedentários.

Na Tabela a seguir apresenta-se a distribuição do risco baixo acompanhado do índice de massa corporal.
Cerca de $70 \%$ dos pesquisados estavam acima do peso. Nos usuários que foram classificados com risco cardiovascular baixo, $65 \%$ apresentaram sobrepeso ou obesidade. Conforme a Tabela 3, 59\% das mulheres que apresentaram bom resultado no EF estão acima do peso; dessas, $20 \%$ são obesas. Nos homens, $86 \%$ apresentaram peso inadequado, e obesos foram quase $29 \%$. Este resultado confirma o que outros autores colocam a respeito da prevalência crescente do sobrepeso e obesidade em diversas populações, incluindo o Brasil. A obesidade é um fator forte de risco cardiovascular independente e está relacionado a hábitos de vida, como o sedentarismo, além de contribuir para o agravo e surgimento de comorbidades (OGDEN et al., 2006; MONTENEGRO NETO et al., 2008; ASSICIAÇÃO..., 2009).

Neste estudo, o risco baixo representou a maioria, porém com fatores de riscos associados que, em longo prazo, podem contribuir para maior morbimortalidade e aumento do RCV com o passar dos anos. A ausência de intervenções e assistência voltadas para a promoção e a prevenção em saúde com incentivo à mudança do estilo de vida, resultará em usuários com RCV aumentado nos próximos dez anos ou menos.

Esse resultado reforça a necessidade de reorganização da Atenção Primária em Saúde (APS), com foco na prevenção e promoção das DCNTs. Sabendo-se que estas doenças são um problema cada vez mais

Tabela 3 - Distribuição do risco cardiovascular baixo e IMC por sexo na população estudada, Palmas - TO -, 2016

\begin{tabular}{|c|c|c|c|c|c|c|c|c|}
\hline \multicolumn{9}{|c|}{ Risco Baixo no Sexo Masculino } \\
\hline Escore em \% & \multicolumn{2}{|c|}{$1-3 \%$} & \multicolumn{2}{|c|}{$4-6 \%$} & \multicolumn{2}{|c|}{$7-9 \%$} & \multicolumn{2}{|c|}{ Total } \\
\hline $\mathrm{n}$ & \multicolumn{2}{|c|}{6} & \multicolumn{2}{|c|}{5} & \multicolumn{2}{|c|}{3} & \multicolumn{2}{|c|}{14} \\
\hline$\%$ & \multicolumn{2}{|c|}{42,9} & \multicolumn{2}{|c|}{35,7} & \multicolumn{2}{|c|}{21,4} & \multicolumn{2}{|c|}{100} \\
\hline IMC & $\mathbf{n}$ & $\%$ & $\mathbf{n}$ & $\%$ & $\mathbf{n}$ & $\%$ & $\mathrm{n} T$ & $\% \mathrm{~T}$ \\
\hline Peso Adequado & 2 & 33,3 & 0 & 0 & 0 & 0 & 2 & 14,3 \\
\hline Sobrepeso & 3 & 50 & 2 & 40 & 3 & 100 & 8 & 57,1 \\
\hline Obesidade & 1 & 16,7 & 3 & 60 & 0 & 0 & 1 & 28,6 \\
\hline \multicolumn{9}{|c|}{ Risco Baixo no Sexo Feminino } \\
\hline Escore em \% & \multicolumn{2}{|c|}{$1-3 \%$} & \multicolumn{2}{|c|}{$4-6 \%$} & \multicolumn{2}{|c|}{$7-9 \%$} & \multicolumn{2}{|c|}{ Total } \\
\hline $\mathrm{N}$ & \multicolumn{2}{|c|}{25} & \multicolumn{2}{|c|}{7} & \multicolumn{2}{|c|}{12} & \multicolumn{2}{|c|}{44} \\
\hline$\%$ & \multicolumn{2}{|c|}{56,8} & \multicolumn{2}{|c|}{15,9} & \multicolumn{2}{|c|}{27,3} & \multicolumn{2}{|c|}{100} \\
\hline IMC & $\mathbf{n}$ & $\%$ & $\mathbf{n}$ & $\%$ & $\mathbf{n}$ & $\%$ & n T & $\% \mathrm{~T}$ \\
\hline Baixo Peso & 1 & 4 & 0 & 0 & 0 & 0 & 1 & 2,3 \\
\hline Peso Adequado & 13 & 52 & 1 & 14 & 3 & 25 & 17 & 38,6 \\
\hline Sobrepeso & 9 & 36 & 4 & 57 & 4 & 33 & 17 & 38,6 \\
\hline Obesidade & 2 & 8 & 2 & 29 & 5 & 42 & 9 & 20,5 \\
\hline
\end{tabular}

Obs.:* IMC: Índice de Massa Corporal. nT: Número Total. \%T: Porcentagem Total.

Fonte: Dados dos autores. 
frequente e com grande impacto na saúde pública, intervir apenas em populações já doentes ou com alto risco não implicaria mudança significativa no panorama geral da população e não influenciaria na tendência de adoecimento cada vez mais precoce (ALMEIDA et al., 2017).

Houve uma porcentagem significativa de portadores de DCNTs, portanto usuários acompanhados pela USF periodicamente. Ainda assim, evidenciou-se uma série de fatores de risco além das próprias comorbidades, como o sedentarismo, o sobrepeso e a obesidade. Cerca de $76 \%$ dos pacientes portadores de alguma DCNT foram classificados como sedentários e $81 \%$ estavam acima do peso.

Segundo Mendes (2011), a fragilidade no modelo de atenção à saúde predominante na APS, o cuidado fragmentado, episódico, reativo e centrado na doença, não atinge efetividade no controle e prevenção das DCNTs, resultando em acúmulo de fatores de risco ao longo dos anos e maior chance de adoecimento futuro, como pode ser observado na amostra estudada.

Em um estudo realizado em Juazeiro do Norte com escolares, onde a média de idade foi de 21 anos, destacou-se a importância da prevenção dos fatores de RCV, pois, mesmo em uma idade em que não se considera que há um risco acrescido, a morbimortalidade por estas causas vem aumentando gradativamente. A tendência do surgimento precoce das DCVs faz das populações mais jovens um alvo importante para a estratificação. Mesmo em situações de baixo risco global, pode-se evidenciar previsões no aparecimento destas doenças, o que pode servir de embasamento para intervenções com foco na promoção e prevenção em saúde (GOMES; MOREIRA, 2014; GUERRA, 2008).

A abordagem integral na assistência é função de todos da equipe multiprofissional. É importante identificar fatores de risco de forma precoce e promover intervenções em tempo oportuno, além de estratificar riscos para melhor direcionamento do cuidado. Ações como estas são fundamentais para o controle e a prevenção das DCNTs/DCVs, buscando melhor qualidade de vida para a população e reduzindo o adoecimento e mortalidade precoce.

\section{CONCLUSÕES}

O perfil de risco cardiovascular da população estudada apresentou-se satisfatório pelo EF, entretanto evidenciou-se a presença de outros fatores de RCV associados que, em longo prazo, podem levar os mesmos ao adoecimento e elevação deste mesmo risco.
$\mathrm{O} E \mathrm{EF}$ pode ser um instrumento utilizado na estratificação para nortear as intervenções programadas pela ESF em âmbito individual e coletivo, porém destaca-se a importância do cuidado integral aos indivíduos com risco absoluto baixo, abordando outros fatores de risco que também influenciam no RCV, atuando, assim, de forma precoce e evitando o aparecimento de DCNT e DCV.

A assistência fragmentada e focada em doenças crônicas ou agudas não é efetiva no controle e prevenção dos fatores de RCV. A abordagem integral do indivíduo ainda saudável é fundamental para a prevenção e a promoção em saúde com foco na redução da morbimortalidade por DCNT e DCV. Mais estudos na área podem contribuir para ampliar o conhecimento a respeito da estratificação de risco, suas potencialidades e fragilidades, principalmente em populações com risco baixo.

Neste estudo houve algumas limitações, como a perda de usuários, por não retornarem com os exames, e a pouca representatividade de homens na amostra.

A partir da análise realizada, sugere-se que a ESF utilize o EF para estratificação de risco de populações adultas como rotina diária de assistência, visto seu auxílio na identificação de vulnerabilidades ao adoecimento. Além disso, que estabeleça ações de promoção e prevenção em saúde para os usuários que apresentarem risco baixo, além do acompanhamento diferenciado à população com riscos elevados, considerando também outros fatores de risco para DCNT/DCV, como emergentes nas ações individuais e coletivas.

\section{REFERÊNCIAS}

ALMEIDA, L. M. et al. Estratégias e desafios da atenção primária à saúde e controle e prevenção da obesidade. Revista Eletrônica Gestão e Saúde, v. 8, n. 1, p. 114-139, 2017.

ASSOCIAÇÃO BRASILEIRA DE ESTUDOS SOBRE OBESIDADE (Abeso). Diretrizes brasileiras de obesidade 2009/2010. AC Farmacêutica, Itapevi, SP, 3. ed., p. 1-85, 2009.

BEATTY, A. et al. Country-Level Decision Making for Control of Chronic Diseases: Workshop Summary. National Academies Press, 2012.

BRASIL. Ministério da Saúde. Secretaria de Atenção à Saúde. Departamento de Atenção Básica. Estratégias para o cuidado da pessoa com doença crônica: hipertensão arterial sistêmica. Brasília, DF: MS, 2013.

. Ministério da Saúde. Vigitel 2015 Saúde Suplementar: vigilância de fatores de risco e proteção para doenças crônicas por inquérito telefônico. Brasília, DF: Agência Nacional de Saúde Suplementar; MS, 2017a. (Recurso eletrônico). 
BRASIL. Ministério da Saúde. Secretaria de Atenção à Saúde. Departamento de Atenção Básica. Prevenção clínica de doenças cardiovasculares, cerebrovasculares e renais. Brasília, DF: MS, 2006.

Ministério da Saúde. Departamento de Atenção Básica - DAB. Teto, credenciamento e implantação das estratégias de agentes comunitários de saúde, saúde da família e saúde bucal. Palmas/TO: DAB, 2017b.

. Ministério da Saúde. Sistema de Informações sobre Mortalidade (SIM/SUS). Óbitos por residência, por município e capítulo CID-10. Município Palmas, Tocantins. Período de 2010 a 2014. Disponível em: <http://tabnet.datasus. gov.br/cgi/tabcgi.exe?sim/cnv/obt10to.def>. Acesso em: 28 nov. 2016.

. Ministério da Saúde. Plano de ações estratégicas para o enfrentamento das doenças crônicas não transmissíveis (DCNT) no Brasil 2011-2022. Brasília, DF: MS, 2011.

. Ministério da Saúde. Conselho Nacional de Saúde. Comissão Nacional de Ética em pesquisa. Resolução no 146 de 12 de dezembro de 2012: Diretrizes e normas regulamentadoras de pesquisas em seres humanos. Brasília, DF: MS, 2012.

GOMES, E. B.; MOREIRA, T. M. M. Estratificação do risco cardiovascular em adultos jovens: relação com pressão arterial, antropometria e achados bioquímicos. Rev. Fund. Care, on-line, v. 6, n. 3, p. 918-928, 2014.

GUERRA, A. Factores de risco cardiovascular na infância de doença com expressão clínica na idade adulta. Acta Pediátrica Portuguesa, v. 39, n. 1, p. 23-29, 2008.

INSTITUTO BRASILEIRO DE GEOGRAFIA ESTATÍSTICA (IBGE). Diretoria de Pesquisas. Coordenação de população e indicadores sociais. 2016a. Disponível em: <http://cidades.ibge. gov.br/xtras/temas. php?lang=\&codmun=172100\&idtema=130\&search=tocantins $\mid$ palmas $\mid$ estimativa-da-populacao-2016>. Acesso em: 26 fev. 2017.

- Diretoria de Pesquisas. Departamento de População e Indicadores Sociais. Tábuas de mortalidade para o Brasil. 2016b. Disponível em: <ftp://ftp.ibge.gov.br/Ta-
buas_Completas_de_Mortalidade/Tabuas_Completas_de_ Mortalidade_2015/xIs/ambos_os_sexos.xIs $>$. Acesso em: 26 fev. 2017.

LOTUFO, P. A. O escore de risco de Framingham para doenças cardiovasculares. Revista Med., São Paulo, v. 87, n. 4, p. 232-237, 2008.

MADEIRA, T. C. S. et al. Depressão em idosos hipertensos e diabéticos no contexto da atenção primária em saúde. Rev. APS, v. 16, n. 4, p. 393-398, 2013.

MENDES, E. V. As redes de atenção à saúde. Brasília: Organização Pan Americana de Saúde, 2011.

MONTENEGRO NETO, A. N. et al. Obesidade, envelhecimento e risco cardiovascular no Brasil: possíveis soluções para problemas atuais. Rev. Saúde Com., v. 4, n. 1, p. 57-63, 2008.

MOURA, E. C. et al. Atenção à saúde dos homens no âmbito da Estratégia Saúde da Família. Ciência \& Saúde Coletiva, v. 19, n. 2, p. 429-438, 2014.

OGDEN, C. L. et al. Prevalence of overweight and obesity in the United States, 1999-2004. Jama, v. 295, n. 13, p. 1.5491.555, 2006.

OLIVEIRA, D. C.; SILVA, L. L. O que pensam os usuários sobre a saúde: representação social do Sistema Único de Saúde. Rev. Enferm. Uerj, Rio de Janeiro, v. 18, n. 1, p. 14-18, 2010. PIMENTA, H. B.; CALDEIRA, A. P. Fatores de risco cardiovascular entre hipertensos assistidos por Equipes de Saúde da Família. Ciência \& Saúde Coletiva, v. 19, n. 6, p. 1.731-1.739, 2014.

SANTOS, P. A.; PINHO, C. P. S. Diabetes Mellitus em pacientes coronariopatas: prevalência e fatores de risco cardiovascular associado. Rev. Bras. Clin. Med., v. 10, n. 6, p. 469-475, 2012.

SCHMIDT, M. I. et al. Health in Brazil 4. Chronic non-communicable diseases in Brazil: burden and current challenges. The Lancet, n. 377, 2011. 